\title{
NEUTROPHIL-LYMPHOCYTE RATIO AND PLATELET-LYMPHOCYTE RATIO AS PREDICTORS OF CORONARY MICROCIRCULATORY DISEASE OCCURRENCE AND OUTCOME IN PATIENTS WITH CHRONIC CORONARY SYNDROME AND NO SIGNIFICANT CORONARY ARTERY STENOSIS
}

10.36740/WLek202012108

\author{
Piotr Szolc', Łukasz Niewiara' , Marta Kawulak' , Kornelia Szkodoń1 , Elżbieta Ostrowska-Kaim ', Marcin Nosal' \\ Marcin Krzanowski ${ }^{3}$, Krzysztof Żmudka ${ }^{1}$, Jacek Legutko ${ }^{1}$, Bartłomiej Guzik ${ }^{1}$ \\ 'DEPARTMENT OF INTERVENTIONAL CARDIOLOGY, JAGIELLONIAN UNIVERSITY MEDICAL COLLEGE, INSTITUTE OF CARDIOLOGY, JOHN PAUL II HOSPITAL, \\ CRACOW, POLAND \\ 2DEPARTMENT OF INVASIVE CARDIOLOGY, ELECTROTHERAPY AND ANGIOLOGY, JOHN PAUL II HOSPITAL, KROSNO, POLAND \\ ${ }^{3}$ DEPARTMENT OF NEPHROLOGY, JAGIELLONIAN UNIVERSITY MEDICAL COLLEGE, CRACOW, POLAND
}

\begin{abstract}
Introduction: Index of microcirculatory resistance assessment is an invasive method of measuring coronary microcirculation function. Association between impaired microcirculatory function and higher rate of cardiovascular events was proven. Neutrophil-lymphocyte ratio and platelet-lymphocyte ratio seem to be a promising parameters to predict coronary microcirculatory disease in patients with chronic coronary syndrome.

The aim: To determine neutrophil-lymphocyte ratio and platelet-lymphocyte ratio levels in patients with coronary microcirculatory disease and potential association with clinical outcome.

Material and methods: 82 consecutive patients with mean age of 67 years, $67 \%$ male, were tested for presence of coronary microcirculatory disease using index of microcirculatory resistance. Neutrophil-lymphocyte ratio and platelet-lymphocyte ratio were calculated based on admission full blood count. Follow-up with major adverse cardiac and cardiovascular events registration was performed (median 24 months).

Results: The study showed significantly higher neutrophil-lymphocyte ratio and platelet-lymphocyte ratio in patients with coronary microcirculatory disease compared to control group ( $3.58 \pm 2.61$ vs $2.54 \pm 1.09$ and $164 \pm 87.9$ vs $124 \pm 36.6$ respectively). Higher level of platelet-lymphocyte ratio in patients with coronary microcirculatory disease results in worse MACCE-free survival. Optimal cut-off values of neutrophil-lymphocyte ratio and platelet-lymphocyte ratio to detect coronary microcirculatory disease were 3.2 and 181.3 , respectively.

Conclusions: Higher neutrophil-lymphocyte ratio and platelet-lymphocyte ratio are associated with increased index of microcirculatory resistance value. Platelet-lymphocyte ratio may be used as a predictor of worse outcome in patients with coronary microcirculatory disease.
\end{abstract}

KEY WORDS: inflammation, coronary artery disease, functional assessment, microcirculation, index of microcirculatory resistance, coronary microcirculatory disease

Wiad Lek. 2020;73(12 p. I):2598-2606

\author{
ABBREVIATIONS: \\ CAD - coronary artery disease \\ CCS - Canadian Cardiovascular Society \\ CMD - coronary microcirculatory disease \\ CVD - cardiovascular disease \\ ETT - exercise treadmill test \\ PLR - platelet-lymphocyte ratio \\ NLR - neutrophil-lymphocyte ratio \\ NA - not applicable \\ FFR - fractional flow reserve \\ CFR - coronary flow reserve \\ IMR - index of microcirculatory resistance \\ SPECT - single photon emission computed tomography \\ PCI - percutaneous coronary intervention
}

CABG - coronary artery bypass grafting

$\mathrm{SD}$ - standard deviation

LVEF - left ventricle ejection fraction

LAD - left anterior descending

LCX - left circumflex branch

RCA - right coronary artery

MACCE - major adverse cardiac and cardiovascular event

\section{INTRODUCTION}

Cardiovascular diseases are leading cause of morbidity and mortality throughout the world. According to the literature about $30 \%$ all deaths worldwide are a result of CVD $[1,2]$. Heart ischemic disease is the most common diagnosis in this wide, 
heterogenic group [3,4]. Cardiac ischemic disease may affect both epicardial arteries and coronary microcirculation which consists of small vessels arterioles and capillaries [5]. Dysfunction of coronary microcirculation is associated with increased risk of major adverse cardiovascular events including cardiac death, stroke, and heart failure [6,7]. Chronic inflammation is considered as an important factor in pathophysiology of atherosclerosis [8-10]. Elevated level of inflammatory markers is observed in disease of epicardial arteries $[11,12]$. Pathogenesis of coronary microcirculatory dysfunction is still understudied and it remains an interesting clinical issue. Some inflammatory indicators are considered to take a part in coronary microcirculation pathology $[12,13]$. The neutrophil-to-lymphocyte ratio (NLR) and platelet-to-lymphocyte ratio (PLR) have been investigated as new markers for cardiovascular prognosis [14-16]. Elevated levels of both, NLR and PLR ratio were observed in patients with acute coronary syndrome as predictors of worse outcome [17]. Neutrophil-to-lymphocyte ratio measured in patients with chronic coronary syndrome is connected with higher severity of coronary artery disease [18]. To our best knowledge there is no data available on NLR and PLR levels according to coronary microcirculatory dysfunction in chronic coronary syndrome. It was shown that microcirculatory dysfunction is connected with higher risk of the cardiovascular events $[5,6]$. The index of microcirculatory resistance (IMR) measurement is an invasive method of coronary microcirculation assessment [19-21].

\section{THE AIM}

The aim of our study is to investigate if the levels of neutrophil-lymphocyte ratio (NLR) and platelet-lymphocyte ratio (PLR) are associated with coronary microcirculation impairment in patients with chronic coronary syndrome. We hypothesize that:

- levels of neutrophil-lymphocyte ratio (NLR) and platelet-lymphocyte ratio (PLR) are elevated in patients with

Table 1. Baseline characteristics.

\begin{tabular}{|c|c|c|c|c|}
\hline & All patients & CMD (IMR<25) & Control group $($ IMR $<25)$ & P-value \\
\hline & $\mathrm{N}=82$ & $\mathbf{N}=\mathbf{3 0}$ & $\mathrm{N}=52$ & \\
\hline Age, mean (SD) [year] & $67.0(8.74)$ & $69.1(9.74)$ & $65.8(7.97)$ & 0.128 \\
\hline Sex male, $\mathrm{n}(\%)$ & $54(66.7)$ & $20(69.0)$ & $34(65.4)$ & 0.935 \\
\hline SBP, mean (SD) $[\mathrm{mmHg}]$ & $130(12.5)$ & $131(11.1)$ & $129(13.3)$ & 0.656 \\
\hline LVEF, mean (SD) [\%] & $55.1(11.2)$ & $53.8(15.1)$ & $55.9(8.08)$ & 0.582 \\
\hline Vessel & & & & $0.027 *$ \\
\hline LAD, n (\%) & $39(47.6)$ & $16(53.3)$ & $23(44.2)$ & \\
\hline LCx, n (\%) & $27(32.9)$ & $5(16.7)$ & $22(42.3)$ & \\
\hline $\mathrm{RCA}, \mathrm{n}(\%)$ & $16(19.5)$ & $9(30.0)$ & $7(13.5)$ & \\
\hline Diabetes, n (\%) & $29(35.4)$ & $9(30.0)$ & $20(38.5)$ & 0.595 \\
\hline Arterial hypertension, $\mathrm{n}(\%)$ & $79(96.3)$ & $30(100)$ & $49(94.2)$ & 0.295 \\
\hline Smoking, n (\%) & $12(16.2)$ & $5(18.5)$ & $7(14.9)$ & 0.749 \\
\hline \multicolumn{5}{|l|}{ Medications: } \\
\hline ASA, n (\%) & $73(89.0)$ & $24(80.0)$ & $49(94.2)$ & 0.068 \\
\hline B-blocker, n (\%) & $65(79.3)$ & $23(76.7)$ & $42(80.8)$ & 0.874 \\
\hline ACEI/ARB, n (\%) & $72(88.9)$ & $25(86.2)$ & $47(90.4)$ & 0.715 \\
\hline Dihydropyridine calcium blocker, n (\%) & $26(31.7)$ & $14(46.7)$ & $12(23.1)$ & $0.049 *$ \\
\hline Loop diuretics, n (\%) & $11(13.6)$ & $6(20.7)$ & $5(9.62)$ & 0.189 \\
\hline Statin, n (\%) & $82(100)$ & $30(100)$ & $52(100)$ & NA \\
\hline
\end{tabular}

SBP - systolic blood pressure; LVEF - left ventricle ejection fraction; LAD - left anterior descending artery; LCx - left circumflex artery; RCA - right coronary artery; ASA - acetylsalicylic acid; b-bloker - beta-adrenolitic drug; $A C E I$ - angiotensin converting enzyme inhibitor; $A R B$ - angiotensyn receptor blocker; * statistical significance $(p<0,05)$

Table 2. Coronary physiology assessment.

\begin{tabular}{ccccc}
\hline & All patients & CMD (IMR $\geq 25)$ & Control group (IMR<25) & P-value \\
\hline & $\mathbf{N}=\mathbf{8 2}$ & $\mathbf{N}=\mathbf{3 0}$ & $\mathbf{N}=\mathbf{5 2}$ & \\
\hline FFR, mean (SD) & $0.88(0.05)$ & $0.87(0.05)$ & $0.88(0.05)$ & 0.281 \\
\hline CFR, mean (SD) & $2.22(1.13)$ & $1.82(0.78)$ & $2.47(1.25)$ & $0.007^{*}$ \\
\hline IMR, mean (SD) & $24.8(15.4)$ & $39.3(15.8)$ & $16.0(5.05)$ & $<0.001^{*}$ \\
\hline
\end{tabular}

FFR - fractional flow reserve; CFR - coronary flow reserve; IMR - index of microcirculatory resistance; * statistical significance $(\mathrm{p}<0,05)$ 
Table 3. Laboratory results

\begin{tabular}{|c|c|c|c|c|}
\hline & All patients & CMD (IMR $\geq 25)$ & Control group (IMR<25) & P-value \\
\hline & $\mathrm{N}=82$ & $\mathbf{N}=\mathbf{3 0}$ & $\mathrm{N}=52$ & \\
\hline NLR, mean (SD) & $2.92(1.86)$ & $3.58(2.61)$ & $2.54(1.09)$ & $0.045 *$ \\
\hline PLR, mean (SD) & $139(63.1)$ & $164(87.9)$ & $124(36.6)$ & $0.023 *$ \\
\hline RBC, mean $(\mathrm{SD})\left[* 10^{6} / \mathrm{uL}\right]$ & $4.70(0.40)$ & $4.70(0.43)$ & $4.70(0.38)$ & 0.974 \\
\hline $\mathrm{HGB}$, mean $(\mathrm{SD})[\mathrm{g} / \mathrm{dL}]$ & $14.2(1.42)$ & $14.1(1.56)$ & $14.2(1.36)$ & 0.841 \\
\hline WBC, mean (SD) [*10\%/uL] & $7.01(1.58)$ & $6.84(1.42)$ & $7.11(1.67)$ & 0.439 \\
\hline PLT, mean (SD) [*10\%/uL] & $222(50.3)$ & $224(63.0)$ & $221(41.8)$ & 0.834 \\
\hline Creatinine, mean (SD) [umol/L] & $83.5(18.9)$ & $88.7(23.7)$ & $80.6(15.0)$ & 0.099 \\
\hline Glucose, mean (SD) [mmol/L] & $6.50(1.85)$ & $6.50(1.77)$ & $6.50(1.92)$ & 0.994 \\
\hline HbA1c, mean (SD) [\%] & $6.03(0.93)$ & $5.69(0.40)$ & $6.20(1.08)$ & $0.031 *$ \\
\hline $\mathrm{LDL}$, mean (SD) [mmol/L] & $2.77(1.20)$ & $2.84(1.29)$ & $2.73(1.16)$ & 0.697 \\
\hline $\mathrm{HDL}$, mean (SD) [mmol/L] & $1.33(0.34)$ & $1.33(0.34)$ & $1.33(0.35)$ & 0.999 \\
\hline Total cholesterol, mean (SD) [mmol/L] & $4.38(1.31)$ & $4.51(1.32)$ & $4.31(1.31)$ & 0.493 \\
\hline TG, mean (SD) $[\mathrm{mmol} / \mathrm{L}]$ & $1.54(0.85)$ & $1.53(0.98)$ & $1.55(0.77)$ & 0.934 \\
\hline
\end{tabular}

LDL - low density lipoprotein; HDL - high density lipoprotein; TG - triglycerides; NLR - neutrophil-lymphocyte ratio; PLR - platelet-lymphocyte ratio; CMD coronary microcirculatory disease; IMR - index of microcirculatory resistance; RBC - red blood count; HGB - haemoglobin; WBC - white blood count; PLT - platelets; HbA1c - glycated haemoglobin;

* statistical significance $(p<0,05)$
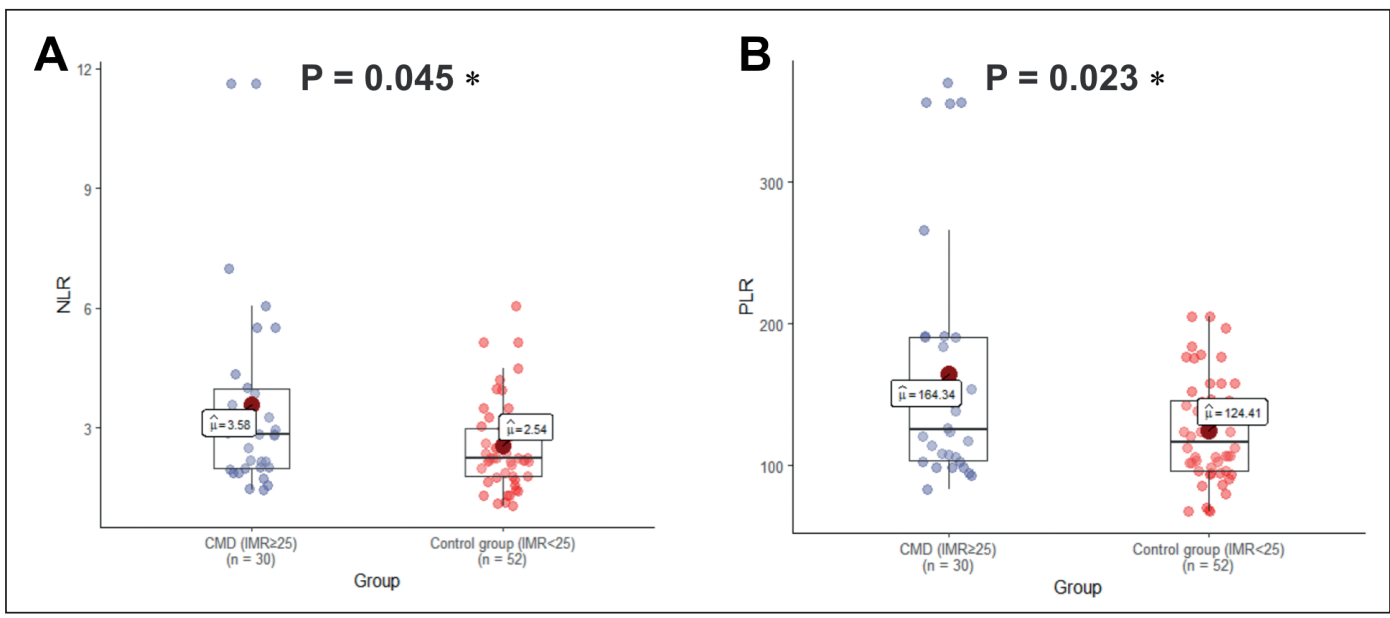

Fig. 1. Comparison of neutrophile-lymphocyte ratio (NLR) (A) and plateletlymphocyte (PLR) (B) value in CMD and control group. NLR - neutrophillymphocyte ratio; PLR - platelet-lymphocyte ratio; CMD coronary microcirculatory disease; IMR - index of microcirculatory resistance; $\bullet$ statistical significance $(p<0,05)$

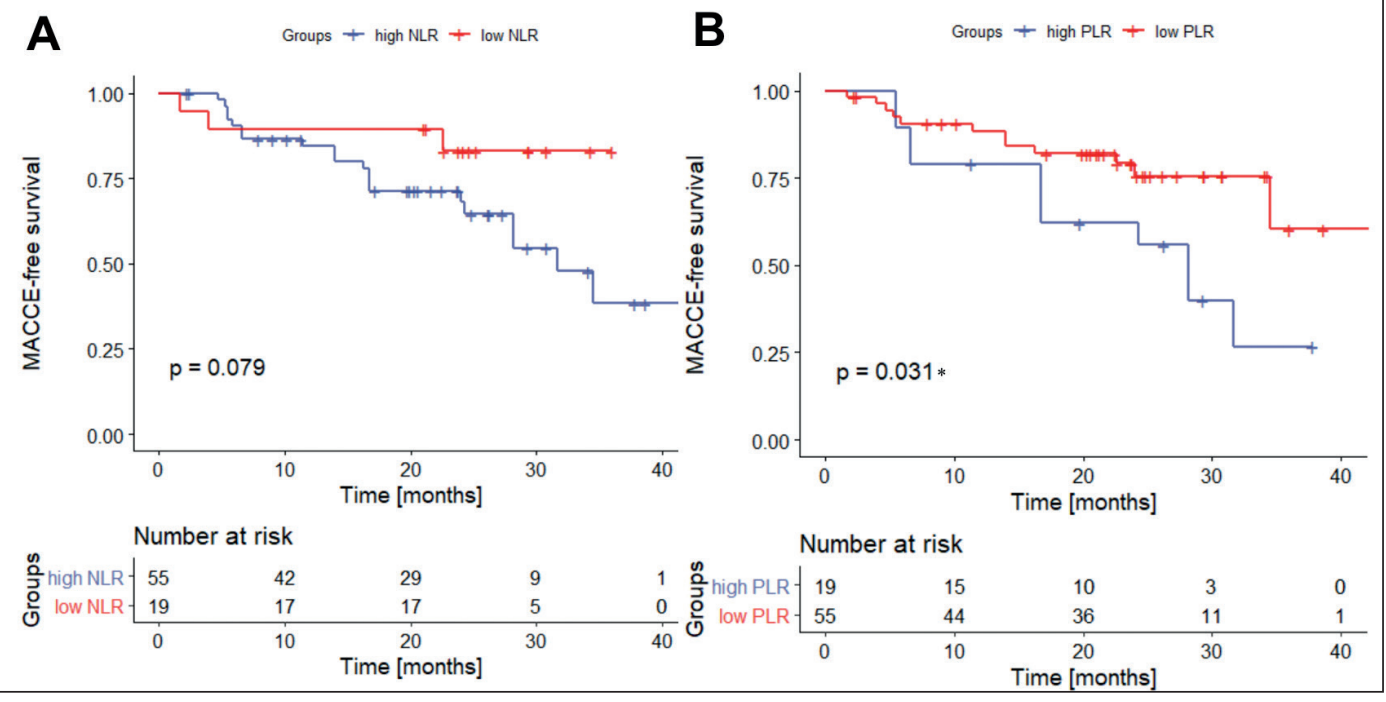

Fig. 2. MACCE free survival in patients with $C M D$ and low/high NLR (A) and PLR (B) levels (KaplanMeier estimator). PLR - platelet-lymphocyte ratio; NLR - neutrophillymphocyte ratio; * statistical significance $(\mathrm{p}<0,05)$ 


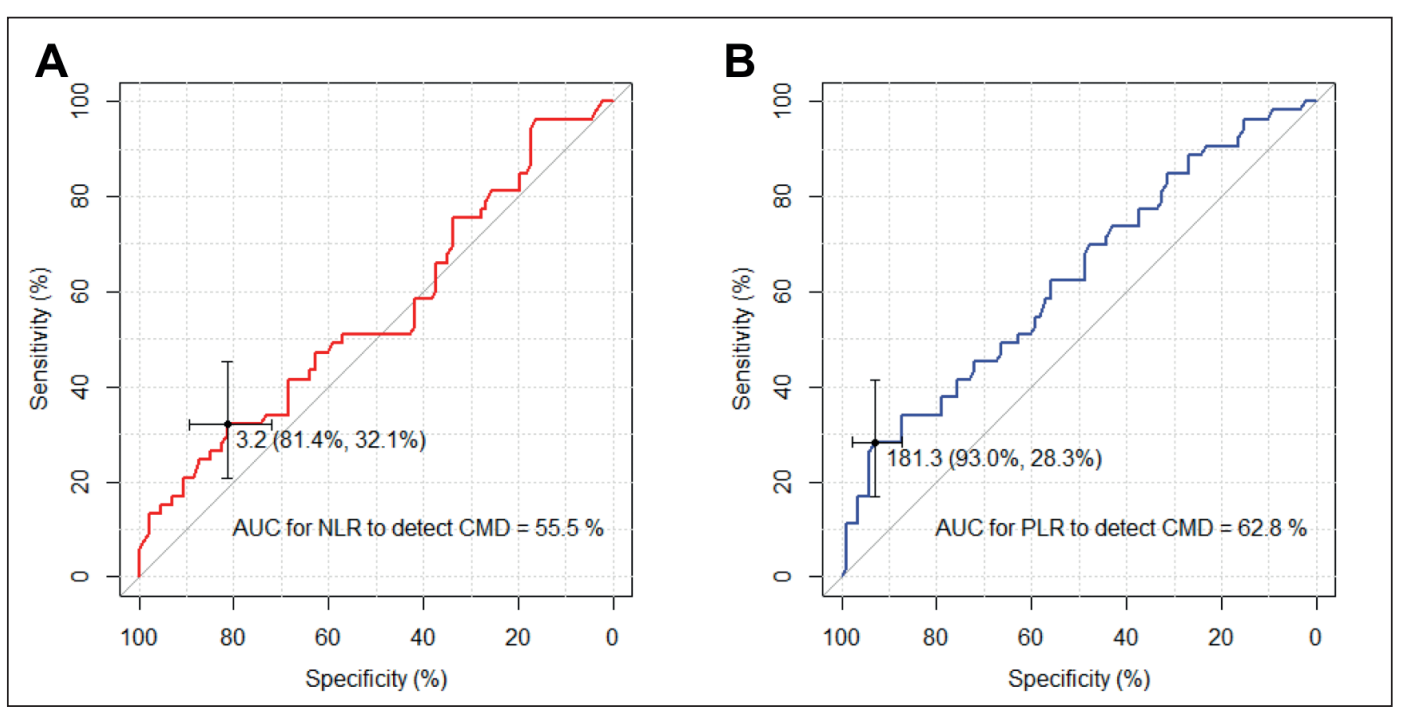

Fig. 3. ROC curves for NLR (A) and PLR (B) to detect CMD. $\mathrm{ROC}$ - receiver operating characterisrtic; PLR-platelet-lymphocyte ratio; NLR - neutrophillymphocyte ratio; $C M D$ coronary microcirculatory disease. chronic coronary syndrome and coronary microcirculatory impairment compared to patients with chronic coronary syndrome and normal microcirculatory function - patients with higher level of neutrophil-lymphocyte ratio (NLR) and platelet-lymphocyte ratio (PLR) have worse survival probability compared to patients with lower NLR and PLR level in group of coronary microcirculatory disease

\section{MATERIAL AND METHODS}

This was a prospective study. We enrolled 82 consecutive, adult patients admitted to The Department of Interventional Cardiology in The John Paul II Hospital, Cracow, Poland. Patients had symptoms of stable angina, grade I - III according to the Canadian Cardiovascular Scale (CCS). Evidence of ischemia was proved with non invasive stress tests: exercise treadmill test (ETT), stress echocardiography or single photon emission computed tomography (SPECT). We excluded patients with diagnosed malignant neoplasm, history of prior percutaneous coronary angioplasty or coronary artery bypass grafting, history of chronic inflammatory disease, infectious disease or active bleeding, expected survival less then 1 year.

Vital sings such as arterial blood pressure, heart rate were collected. Risk factors including arterial hypertension, diabetes, smoking were recorded. We gathered information about current medical treatment. Blood samples $(2 \mathrm{ml})$ were taken to test-tubes with EDTA (full blood count measurement) and test-tubes with no anticoagulant (chemistry tests). Peripheral full blood count was analyzed Coulter optical measurement system (Beckman Coulter, United States). Neutrophil-lymphocyte ratio (NLR) and platelet-lymphocyte ratio (PLR) were counted. In cardiac ultrasound (Philips XC50, Holland) left ventricle ejection fraction was measured with Simpson's biplane method.

The patients were screened for presence of intermediate stenosis of epicardial arteries. We considered coronary artery stenosis of $50-90 \%$ as a borderline lesion [22]. If present, we performed full coronary physiology assessment using Pres- sure Wire X (Abbott Vascular, Santa Clara, California, US) connected to computer by Wi-Fi. Measurements of mean transit time were initially made in resting conditions. Than we induced maximal hyperemia with continuous intravenous infusion of adenosine $(140 \mathrm{ug} / \mathrm{kg} / \mathrm{min})$ and repeated transit mean time registration. FFR was calculated as a ratio between blood pressure in distal part of examined coronary artery and blood pressure in aorta at maximal hyperemia. Result of ratio between resting and hyperemic transit mean time was CFR. IMR value was calculated by multiplying mean transit time of room temperature saline through coronary artery and distal coronary artery pressure. All measurements registered during procedure were automatically sent to Coroflow software (Coroventis AB, Uppsala, Sweden) which performed calculations of fractional flow reserve (FFR), coronary flow reserve (CFR) and index of microcirculatory resistance (IMR) [23]. Patients with positive result of fractional flow reserve (FFR 0.8) underwent coronary revascularization and were excluded from this study. If more than one vessel was examined in one patient, results from all vessels were analyzed. The study protocol received positive opinion from Jagiellonian University Ethical Committee (nr 122.6120.262.2015).

\section{FOLLOW-UP}

Clinical follow was made at 24 months. Major adverse cardiac and cardiovascular event (MACCE) as a primary composite end point was assessed, which was defined as myocardial infarction, percutaneous coronary angioplasty, stroke, all cause-death.

\section{STATISTICAL METHODS}

Statistical analysis was performed using IBM SPSS Statistics (version 25) and $\mathrm{R}$ language (R-Studio, version 8.9) packages for Windows. Categorical data were described as percentages, while continuous data were described as mean $( \pm S D)$. Normal distribution was tested by Kolmogorov-Smirnov test. Categorical variables were compared using chi-squared test. Continuous 
Table 4. Logistic regression models.

\begin{tabular}{ccccccc}
\hline & \multicolumn{3}{c}{ NLR } & & PLR & Cl \\
\hline Predictors & Odds Ratios & $\mathbf{C l}$ & $\mathbf{p}$ & Odds Ratios & $\mathbf{P}$ \\
\hline (Intercept) & 0.16 & $0.03-0.78$ & $0.023^{*}$ & 0.05 & $0.01-0.48$ & $0.010^{*}$ \\
\hline NLR increase of 1 unit & 1.50 & $1.05-2.13$ & $0.026^{*}$ & & & \\
\hline Present smoker & 1.54 & $0.35-6.70$ & 0.563 & 2.07 & $0.47-9.12$ & 0.338 \\
\hline Diabetes & 0.84 & $0.27-2.59$ & 0.762 & 0.84 & $0.27-2.57$ & 0.756 \\
\hline Male sex & 1.03 & $0.32-3.35$ & 0.959 & 1.57 & $0.47-5.26$ & 0.461 \\
\hline PLR increase of 10 units & & & & 1.16 & $1.03-1.31$ & $\mathbf{0 . 0 1 6 *}$ \\
\hline
\end{tabular}

NLR - neutrophil-lymphocyte ratio; PLR - platelet-lymphocyte ratio;

* statistical significance $(p<0,05)$

Table 5. Supplementary table. Baseline characteristics.

\begin{tabular}{|c|c|c|c|c|c|c|}
\hline & All patients & IMR- CFR- & IMR- CFR+ & IMR+ CFR- & IMR+ CFR + & P-value \\
\hline & $\mathrm{N}=76$ & $\mathrm{~N}=32$ & $\mathrm{~N}=15$ & $\mathrm{~N}=7$ & $\mathrm{~N}=\mathbf{2 2}$ & \\
\hline Age, mean (SD) [year] & $67.4(8.65)$ & $66.6(8.06)$ & $66.6(7.76)$ & $67.6(9.57)$ & $69.1(10.0)$ & 0.738 \\
\hline Sex male, n (\%) & $49(65.3)$ & $16(50.0)$ & $13(86.7)$ & $6(85.7)$ & $14(66.7)$ & 0.055 \\
\hline SBP, mean (SD) [mmHg] & $130(12.1)$ & $128(12.9)$ & $134(13.1)$ & $132(10.5)$ & $131(10.8)$ & 0.500 \\
\hline LVEF, mean (SD) [\%] & $54.8(11.5)$ & $55.8(8.05)$ & $54.8(9.74)$ & $60.0(15.4)$ & $51.6(14.9)$ & 0.540 \\
\hline Vessel: & & & & & & 0.023 \\
\hline LAD, n (\%) & $36(47.4)$ & $17(53.1)$ & $5(33.3)$ & $5(71.4)$ & $9(40.9)$ & \\
\hline LCx, n (\%) & $24(31.6)$ & $10(31.2)$ & $9(60.0)$ & $0(0.00)$ & $5(22.7)$ & \\
\hline RCA, n (\%) & $15(19.7)$ & $5(15.6)$ & $1(6.67)$ & $1(14.3)$ & $8(36.4)$ & \\
\hline Diabetes, n (\%) & $27(35.5)$ & $11(34.4)$ & $8(53.3)$ & $2(28.6)$ & $6(27.3)$ & 0.419 \\
\hline Arterial hypertension, n (\%) & $74(97.4)$ & $30(93.8)$ & $15(100)$ & $7(100)$ & $22(100)$ & 0.753 \\
\hline Smoking, n (\%) & $12(17.1)$ & $4(13.3)$ & $3(21.4)$ & $1(14.3)$ & $4(21.1)$ & 0.899 \\
\hline \multicolumn{7}{|l|}{ Medications: } \\
\hline ASA, n (\%) & $68(89.5)$ & $29(90.6)$ & $15(100)$ & $6(85.7)$ & $18(81.8)$ & 0.311 \\
\hline B-blocker, n (\%) & $61(80.3)$ & $28(87.5)$ & $11(73.3)$ & $5(71.4)$ & $17(77.3)$ & 0.521 \\
\hline ACEI/ARB, n (\%) & $66(88.0)$ & $28(87.5)$ & $14(93.3)$ & $5(71.4)$ & $19(90.5)$ & 0.515 \\
\hline Dihydropyridine calcium blocker, n (\%) & $26(34.2)$ & $9(28.1)$ & $3(20.0)$ & $2(28.6)$ & $12(54.5)$ & 0.126 \\
\hline Loop diuretics, n (\%) & $10(13.3)$ & $3(9.38)$ & $2(13.3)$ & $1(14.3)$ & $4(19.0)$ & 0.769 \\
\hline Statin, n (\%) & $76(100)$ & $32(100)$ & $15(100)$ & $7(100)$ & $22(100)$ & NA \\
\hline
\end{tabular}

SBP - systolic blood pressure; LVEF - left ventricle ejection fraction; LAD - left anterior descending artery; LCX - left circumflex artery; RCA - right coronary artery; $A S A$ - acetylsalicylic acid; b-bloker - beta-adrenolitic drug; ACEI - angiotensin converting enzyme inhibitor; ARB - angiotensyn receptor blocker; ${ }^{*}$ statistical significance $(p<0,05)$

Table 6. Supplementary table. Coronary physiology assessment.

\begin{tabular}{|c|c|c|c|c|c|c|}
\hline & All patients & IMR- CFR- & IMR- CFR+ & IMR+ CFR- & IMR + CFR + & P-value \\
\hline & $N=76$ & $\mathrm{~N}=32$ & $N=15$ & $N=7$ & $\mathrm{~N}=\mathbf{2 2}$ & \\
\hline FFR, mean (SD) & $0.88(0.05)$ & $0.89(0.05)$ & $0.88(0.05)$ & $0.86(0.04)$ & $0.87(0.05)$ & 0.474 \\
\hline CFR, mean (SD) & $2.22(1.13)$ & $3.02(1.14)$ & $1.30(0.28)$ & $2.99(0.55)$ & $1.45(0.36)$ & $<0.001^{*}$ \\
\hline IMR, mean (SD) & $25.1(15.4)$ & $16.2(4.95)$ & $16.3(5.07)$ & $31.5(2.99)$ & $41.9(17.5)$ & $<0.001^{*}$ \\
\hline
\end{tabular}

FFR - fractional flow reserve; CFR - coronary flow reserve; IMR - index of microcirculatory resistance; ${ }^{*}$ statistical significance $(p<0,05)$

variables were compared by Mann-Whitney or t-student tests. The optimal cut off point for multiple continuous variables was determined using the maximally selected rank statistics from the 'maxstat' R package. Survival probability was evaluated using Kaplan-Meier survival estimators. A value of $\mathrm{P}<0.05$ was established as statistical significant. Logistic mixed effects regression models were built, using NLR, PLR and common atherosclerosis risk factors (i.e. diabetes, smoking status, sex) 
Table 7. Supplementary table. Laboratory results.

\begin{tabular}{|c|c|c|c|c|c|c|}
\hline & All patients & IMR- CFR- & IMR- CFR+ & IMR+ CFR- & IMR+ CFR + & P-value \\
\hline & $\mathrm{N}=76$ & $\mathrm{~N}=32$ & $\mathrm{~N}=15$ & $N=7$ & $\mathrm{~N}=\mathbf{2 2}$ & \\
\hline NLR, mean (SD) & 2.28 & 2.34 & 2.14 & 2.02 & 2.82 & 0.220 \\
\hline PLR, mean (SD) & 119 & 112 & 107 & 117 & 132 & 0.161 \\
\hline $\mathrm{RBC}$, mean $(\mathrm{SD})\left[* 10^{6} / \mathrm{uL}\right]$ & $4.69(0.39)$ & $4.64(0.41)$ & $4.74(0.29)$ & $4.52(0.46)$ & $4.78(0.40)$ & 0.352 \\
\hline $\mathrm{HGB}$, mean $(\mathrm{SD})[\mathrm{d} / \mathrm{dL}]$ & $14.2(1.44)$ & $14.0(1.47)$ & $14.4(1.19)$ & $13.9(1.20)$ & $14.3(1.64)$ & 0.704 \\
\hline WBC, mean (SD) [*10\%/uL] & $6.95(1.60)$ & $7.31(1.85)$ & $6.34(1.16)$ & $6.98(1.27)$ & $6.83(1.51)$ & 0.273 \\
\hline PLT, mean (SD) [*10\%/uL] & $222(51.0)$ & $224(42.1)$ & $214(42.9)$ & $222(56.9)$ & $226(66.7)$ & 0.907 \\
\hline Creatinine, mean (SD) [umol/L] & $83.9(19.4)$ & $82.4(16.7)$ & $78.4(12.9)$ & $86.9(11.2)$ & $88.8(27.1)$ & 0.407 \\
\hline Glucose, mean (SD) [mmol/l] & $6.58(1.89)$ & $6.39(1.89)$ & $7.21(2.08)$ & $6.63(1.51)$ & $6.43(1.90)$ & 0.578 \\
\hline $\mathrm{HbA} 1 \mathrm{c}$, mean (SD) [\%] & $6.06(0.95)$ & $6.27(0.91)$ & $6.25(1.41)$ & $5.77(0.42)$ & $5.65(0.42)$ & 0.316 \\
\hline LDL, mean (SD) [mmol/L] & $2.72(1.19)$ & $2.75(1.28)$ & $2.47(0.69)$ & $2.17(0.83)$ & $3.02(1.38)$ & 0.307 \\
\hline $\mathrm{HDL}$, mean (SD) [mmol/L] & $1.31(0.32)$ & $1.28(0.32)$ & $1.31(0.30)$ & $1.26(0.37)$ & $1.36(0.34)$ & 0.841 \\
\hline Tchol, mean (SD) [mmol/L] & $4.32(1.27)$ & $4.27(1.39)$ & $4.06(0.78)$ & $3.77(0.94)$ & $4.73(1.39)$ & 0.234 \\
\hline $\mathrm{TG}$, mean (SD) $[\mathrm{mmol} / \mathrm{L}]$ & $1.57(0.87)$ & $1.68(0.82)$ & $1.41(0.72)$ & $1.43(0.37)$ & $1.58(1.13)$ & 0.766 \\
\hline
\end{tabular}

LDL - low density lipoprotein; HDL - high density lipoprotein; TG - triglycerides; NLR - neutrophil-lymphocyte ratio; PLR - platelet-lymphocyte ratio; CMD coronary microcirculatory disease; IMR - index of microcirculatory resistance; RBC - red blood count; HGB - haemoglobin; WBC - white blood count; PLT - platelets; HbA1c - glycated haemoglobin;

* statistical significance $(p<0,05)$

Table 8. Supplementary table. Follow-up endpoints.

\begin{tabular}{|c|c|c|c|c|}
\hline & All patients & CMD $($ IMR $<U+2265>25)$ & Control group (IMR<25) & p-value \\
\hline & $\mathrm{N}=\mathbf{8 0}$ & $\mathrm{N}=\mathbf{3 0}$ & $\mathrm{N}=50$ & \\
\hline Hospitalization, n (\%) & $25(31.2)$ & $8(26.7)$ & $17(34.0)$ & 0.663 \\
\hline CVD hospitalization, n (\%) & $19(23.8)$ & $5(16.7)$ & $14(28.0)$ & 0.378 \\
\hline AMI, n (\%) & $6(8.45)$ & $2(6.90)$ & $4(9.52)$ & 1.000 \\
\hline TVR, n (\%) & $6(10.3)$ & $2(8.33)$ & $4(11.8)$ & 1.000 \\
\hline MACCE, n (\%) & $24(30.0)$ & $8(26.7)$ & $16(32.0)$ & 0.801 \\
\hline
\end{tabular}

CVD hospitalization - cardiovascular disease hospitalization; AMI - acute myocardial infarction; TVR - target vessel revascularization; MACCE - major adverse cardiac and cardiovascular event

as fixed factors and type of tested vessel as random factor. This approach was used as flow in coronary arteries varies according to type of vessel (i.e. right vs left coronary artery) and the area of myocardium supplied by particular vessel [24].

\section{RESULTS}

The study population consists of 82 consecutive patients who underwent elective coronary angiography in chronic coronary syndrome and did not meet exclusion criteria. Whole population was divided into 2 groups according to result of the index of microcirculatory resistance. Patients included to group of normal coronary microcirculatory function (control group, 52 objects) had IMR $<25$. Patients in coronary microcirculatory dysfunction group (CMD group, 30 objects) had IMR $\geq 25$. Baseline characteristics was shown in table 1. Age distribution in both groups was similar (69.1 \pm 9.74 vs $65.8 \pm 7.97$ years). Number of male patients did not differ in both groups (69\% vs $65 \%)$. There was no significant difference in systolic blood pressure
$(131 \pm 11.1 \mathrm{mmHg}$ vs $129 \pm 13.3 \mathrm{mmHg})$ and left ventricle ejection fraction ( $53.8 \pm 15.1 \%$ vs $55.9 \pm 8 \%$ ) between both groups. 52 main coronary vessels in control group and 30 vessels in CMD group were assessed, while the left anterior descending artery was the most often examined vessel. Both groups did not differ significantly in risk factors of coronary artery disease and taken medications despite dihydropyridine calcium channel blockers which were used more often in CMD group ( $46.7 \%$ vs $23.1 \%$ ) (Table 1 ).

Fractional flow reserve (FFR) result was similar in both groups $(0.87 \pm 0.05$ vs $0.88 \pm 0.05)$. Coronary flow reserve (CFR) was significantly higher in control group compared to CMD group $(2.47 \pm 1.25$ vs $1.82 \pm 0.78 ; \mathrm{P}=0.007)$. The result of index of microcirculatory resistance was criterion of groups distinction as we mentioned previously and it was significantly higher in $\mathrm{CMD}$ group $(39.3 \pm 15.8$ vs $16.0 \pm 5.05 ; \mathrm{P}<0.001)$ (Table 2 ).

Level of serum low-density-lipoprotein (LDL) was lower in control group $(2.84 \pm 1.29$ vs $2.73 \pm 1.16 \mathrm{mmol} / \mathrm{l})$ however difference was not statistically significant $(\mathrm{P}>0.05)$. The results of neutrophil-lymphocyte ratio (NLR) and 
platelet-lymphocyte ratio (PLR) were significantly higher in CMD group (respectively $3.58 \pm 2.61$ vs $2.54 \pm 1.09$; $\mathrm{P}$ $=0.045$ for NLR and $164 \pm 87.9$ vs $124 \pm 36.6 ; \mathrm{P}=0.023$ for PLR) (Table 3, Fig. 1).

Median length of follow-up in whole studied population was 24 months, 22.9 months in CMD group and 24.6 months in control group.

Based on mixed effects logistic regression the increased NLR or PLR values were associated with increased probability od microcirculatory dysfunction, with OR 1.5 (95\% CI: $1.05-2.13$; P < 0.05) and 1.16 (95\% CI $1.03-1.31$; P $<0.05$ ), respectively (Table 4 ) [25].

Kaplan-Meier estimation showed that MACCE-free survival in patient with high PLR level is worse compared to patients with low PLR level in group of coronary microcirculatory disease $(\mathrm{P}=0.031)$ (Fig. $2 \mathrm{~A})$. There is no significant difference in MACCE-free survival between patients with high and low level of NLR in group of coronary microcirculatory disease $(\mathrm{P}=0.079)$, however trend is visible (Fig. 2B).

Study population was divided into 4 groups based on IMR and CFR normal and abnormal results: IMR-CFR-, IMR-CFR+, IMR+CFR-, IMR+CFR+. No significant differences between those groups was founded (Table 5-7.)

Area under curve (AUC) for NLR to detect coronary microcirculatory disease is $55.5 \%$ with cut-off 3.2 and specificity of $81.4 \%$ (Fig. 3A).

Similarly AUC for PLR to detect coronary microcirculatory disease is $62.8 \%$ with cut-off 181.3 and specificity of $93 \%$ (Fig. 3B).

\section{DISCUSSION}

Epicardial coronary arteries visible in angiography are just about $1 \%$ of whole coronary circulation. Nearly $99 \%$ is coronary microcirculation which is not visible in standard angiography, as it consists of vessels with lumen diameter less than $500 \mu \mathrm{m}$ [5]. Epicardial coronary disease as well as disease of coronary microcirculation may be a cause of angina symptoms and a relevant impact on adverse cardiovascular events including cardiac death, stroke, and heart failure $[5,6]$. Prevalence of coronary microcirculatory disease (CMD) is estimated in literature for up to $40 \%$ in patients with suspected coronary artery disease [26]. Guidelines of The European Society of Cardiology for chronic coronary syndrome recommend functional assessment in patients with intermediate coronary artery stenosis (50-90\%) [22]. In case of insignificant fractional flow reserve result (i.e. FFR $>0.8$ ), coronary flow reserve and index of microcirculatory resistance should be measured [22]. Abnormal CFR and IMR results are a basis for diagnosis of coronary microcirculatory disease $[20,21,27]$. Management of CMD differs from treatment of typical epicardial coronary artery disease [22]. In CMD treatment more emphasis is put on vasodilator medications such as calcium channel blockers and long acting nitrates $[27,28]$. On the other hand specific treatment for CMD is still underdeveloped and this topic requires further investigation. According to CORMICA trial detailed diagnostics in patient with coronary artery disease led to better treatment results, as in that study an invasive diagnostics in patients with chronic coronary syndrome did not stop on coronary angiography only. Patients have also undergone complex functional assessment of coronary circulation including provocative test with acetylcholine to detect artery overreactivity. Using this approach coronary microvascular angina or vasospastic angina were diagnosed and appropriate medications were advised. As a result significant reduction of angina symptoms, increase of exertion tolerance and improvement of quality of life were observed as compared with control group, in which only coronary angiography was performed [29].

In our study we propose evaluation of novel inflammatory markers, the neutrophil-lymphocyte ratio (NLR) and platelet-lymphocyte ratio (PLR) as a tool useful in process of qualification of patients with coronary artery disease to complex invasive diagnostics of coronary circulation. NLR and PLR have good specificity to exclude coronary microcirculatory disease, however sensitivity to detect CMD remains low. Even though, utilization of those parameters might help clinicians to select a group of patients with indications for complex coronary circulation assessment, functional examination and provocative testing.

Further investigation is needed to explore possible correlation between NLR and PLR with other, more specific inflammatory markers assessed in patients with coronary microvascular disease.

\section{STUDY LIMITATIONS}

This is single-centre study, whole population was enrolled in one cardiology department. We make an effort so as patients were referred to our department by different physicians. Despite patients were diagnosed and treated by few separate teams, whole group followed the same protocol described in methodology chapter. The population finally included and analyzed was not large. We considered different clinical situations and diseases that may affect result of full blood count. Possible fluctuations of lymphocyte and neutrophil counts may be result of asymptomatic infections. Numerous disorders might be a reason for thrombocytopenia. Decreased thrombocyte production, i.e. ferrum/folic acid/ vitamin B12 deficiency, bone marrow diseases, congenital thrombocytopenia or increased thrombocyte destruction, i.e. hemolysis and side effects of medications. Active bleeding, alcohol addiction, chronic inflammatory diseases may result in thrombocytosis. Every patient with diagnosis that may affect result of white count or platelet count was excluded from this study. In our study correlation between PLR, NLR and other more specific inflammatory indicators was not tested.

\section{CONCLUSIONS}

In this study we observed higher level of inflammatory markers: neutrophil-lymphocyte ratio (NLR) and platelet-lymphocyte ratio (PLR) in patients with chronic coronary syndrome and elevated index of microcirculatory resistance, as com- 
pared to patients with normal IMR. Neutrophil-lymphocyte ratio (NLR) and platelet-lymphocyte ratio (PLR) might be concerned as predictors of coronary microcirculatory disease in chronic coronary syndrome and no significant epicardial stenosis. Assessment of NLR and PLR may be used to select patients who particularly demands complex coronary functional examination during invasive diagnostics.

What is more, higher levels of NLR and PLR are associated with worse MACCE-free survival in this group. Further investigation is needed in aim to compare neutrophil-lymphocyte ratio (NLR) and platelet-lymphocyte ratio (PLR) with other, more specific inflammatory markers of CMD.

\section{REFERENCES}

1. Townsend N, Wilson L, Bhatnagar P, Wickramasinghe K, Rayner M, Nichols M. Cardiovascular disease in Europe: Epidemiological update 2016. Eur Heart J 2016;37;(42):3232-45.

2. Roth GA, Forouzanfar MH, Moran AE, et al. Demographic and epidemiologic drivers of global cardiovascular mortality. N Engl J Med 2015;372;(14):1333-41.

3. Roth GA, Johnson C, Abajobir A, et al. Global, Regional, and National Burden of Cardiovascular Diseases for 10 Causes, 1990 to 2015. J Am Coll Cardiol 2017;70;(1):1-25.

4. Piątek J, Kędziora A, Konstanty-Kalandyk J, et al. Minimally invasive coronary artery bypass as a safe method of surgical revascularization. The step towards hybrid procedures. Adv Interv Cardiol. 2017;13(4):320-325.

5. Fonseca DA, Antunes PE, Cotrim MD. The Morphology, Physiology and Pathophysiology of Coronary Microcirculation. Intechopen, 2016, pp. 15 -47.doi: 10.5772/64537

6. Pries AR, Reglin B. Coronary microcirculatory pathophysiology: can we afford it to remain a black box? Eur Heart J. 2017;38(7):478-488.

7. Taqueti VR, Di Carli MF. Coronary Microvascular Disease Pathogenic Mechanisms and Therapeutic Options: JACC State-of-the-Art Review. J Am Coll Cardiol 2018;72;(21):2625-41.

8. Guzik TJ, Touyz RM. Oxidative stress, inflammation, and vascular aging in hypertension. Hypertension 2017;70;(4):660-7.

9. Raggi P, Genest J, Giles JT, et al. Role of inflammation in the pathogenesis of atherosclerosis and therapeutic interventions. Atherosclerosis 2018;276:98-108.

10. Konduracka E, Niewiara L, Guzik B, et al. Effect of short-term fluctuations in outdoor air pollution on the number of hospital admissions due to acute myocardial infarction among inhabitants of Krakow, Poland. Pol Arch Intern Med. 2019;129(2):884-892.

11. Ali M, Girgis S, Hassan A, Rudick S, Becker RC. Inflammation and coronary artery disease: From pathophysiology to Canakinumab AntiInflammatory Thrombosis Outcomes Study (CANTOS). Coron Artery Dis 2018;29;(5):429-37.

12. Krzanowski M, Krzanowska K, Gajda M, et al. Asymmetric dimethylarginine as a useful risk marker of radial artery calcification in patients with advanced kidney disease. Pol Arch Intern Med. 2018;128(3):157-165.

13. Ding JW, Zhou T, Zheng XX, et al. Effects of High Mobility Group Box-1 Protein on Peripheral Treg/Th17 Balance in Patients with Atherosclerosis. Acta Cardiol Sin. 2018;34(5):399-408.

14. Turcato $G$, Sanchis-Gomar F, Cervellin $G$, et al. Evaluation of neutrophil-lymphocyte and platelet-lymphocyte ratios as predictors of 30-day mortality in patients hospitalized for an episode of acute decompensated heart failure. J Med Biochem 2019;38;(4):452-60.
15. Afari ME, Bhat T. Neutrophil to lymphocyte ratio (NLR) and cardiovascular diseases: An update. Expert Rev Cardiovasc Ther 2016;14;(5):573-7.

16. KaragozI,Yoldas H. Platelet to lymphocyte and neutrophil to lymphocyte ratios as strong predictors of mortality in intensive care population. Rev Assoc Med Bras 2019;65;(5):633-6.

17. Maimaiti A, LiY, Wang YT, et al. Association of platelet-to-lymphocyte count ratio with myocardial reperfusion and major adverse events in patients with acute myocardial infarction: A two-centre retrospective cohort study. BMJ Open 2019;9;(9):e025628.

18. Sari I, Sunbul M, Mammadov C, Durmus E, Bozbay M, Kivrak T, et al. Relation of neutrophil-to-lymphocyte and platelet-to-lymphocyte ratio with coronary artery disease severity in patients undergoing coronary angiography. Kardiol Pol 2015;73;(12):1310-6.

19. Yong AS, Layland J, Fearon WF, et al. Calculation of the index of microcirculatory resistance without coronary wedge pressure measurement in the presence of epicardial stenosis. JACC Cardiovasc Interv 2013;6; (1):53-8.

20. Ng MKC, Yeung AC, Fearon WF. Invasive Assessment of the Coronary Microcirculation Superior Reproducibility and Less Hemodynamic Dependence of Index of Microcirculatory Resistance Compared with Coronary Flow Reserve. Circulation. 2006;113;(17):2054-61.

21. Fearon WF, Kobayashi Y. Invasive Assessment of the Coronary Microvasculature: The Index of Microcirculatory Resistance. Circ Cardiovasc Interv 2017;10;(12).

22. 2019 ESC Guidelines for the diagnosis and management of chronic coronary syndromes. Eur Heart J. 2020;41(3):407-477.

23. Jerabek S, Kovarnik T. Technical aspects and limitations of fractional flow reserve measurement. Acta Cardiol 2019;74;(1):9-16.

24. Bates D, Mächler M, Bolker B, Walker S. Fitting Linear Mixed-Effects Models Using Ime4. Journal of Statistical Software. 2015;67;(1):1-48.

25. Venables WN, Ripley BD. Modern Applied Statistics with S. 4th edn. Springer, 2002.

26. Lüscher TF. From epicardial coronary arteries to the coronary microcirculation: novel insights into a neglected issue. Eur Heart J. 2017;38;(7):461-64.

27. Marinescu MA, Löffler Al, Ouellette M, Smith L, Kramer CM, Bourque JM. Coronary microvascular dysfunction, microvascular angina, and treatment strategies. JACC Cardiovasc Imaging 2015;8;(2):210-20.

28. Ong P, Athanasiadis A, Sechtem U. Pharmacotherapy for coronary microvascular dysfunction. Eur Heart J - Cardiovasc Pharmacother 2015;1;(1):65-71.

29. Ford TJ, Stanley B, Good R, Rocchiccioli P, McEntegart M, Watkins S, et al. Stratified Medical Therapy Using Invasive Coronary Function Testing in Angina: The CorMicA Trial. J Am Coll Cardiol 2018;72;(23):2841-55.

\section{ORCID and contributionship}

Piotr Szolc - 0000-0001-6883-8633 C-D

Łukasz Niewiara - 0000-0001-9652-165X ${ }^{B-C}$

Marta Kawulak - 0000-0002-6259-8576 ${ }^{B}$

Kornelia Szkodoń - 0000-0002-5147-8712 ${ }^{B}$

Elżbieta Ostrowska-Kaim - 0000-0002-7982-7515 ${ }^{\text {B-C }}$

Marcin Nosal - 0000-0003-4435-1219 A,C

Marcin Krzanowski - 0000-0001-7051-6676 C,E

Krzysztof Żmudka - 0000-0001-7429-2825 ${ }^{\text {E-F }}$

Jacek Legutko - 0000-0002-2945-3674 ${ }^{E-F}$

Bartłomiej Guzik - 0000-0002-0630-8394 A,E-F 


\section{Conflict of interest}

Authors declare no conflict of interest.

\section{CORRESPONDING AUTHOR}

\section{Bartłomiej Guzik}

Department of Interventional Cardiology,

Jagiellonian University Medical College,

Institute of Cardiology,

John Paul II Hospital

Prądnicka 80, 31-202, Kraków, Poland

tel.: +48501603091

e-mail: b.guzik@uj.edu.pl

Received: 25.10 .2020

Accepted: 03.12.2020

A - Work concept and design, B - Data collection and analysis, C - Responsibility for statistical analysis,

D-Writing the article, $\mathbf{E}$-Critical review, $\mathbf{F}$ - Final approval of the article 